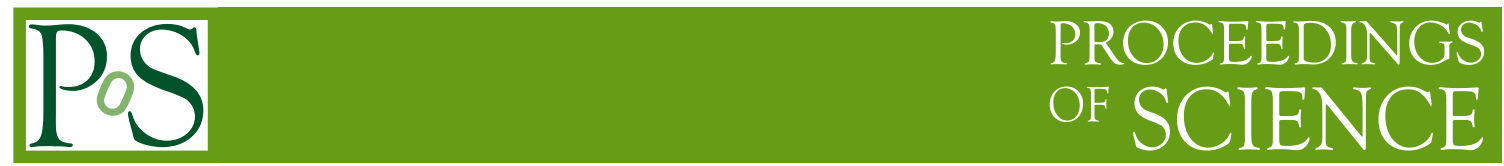

\title{
Measurement of emittance and emittance reduction in MICE
}

\author{
Vassil VERGUILOV*† \\ DPNC - University of Geneva \\ E-mail: vassil.verguilovecern.ch
}

The Muon Ionization Cooling Experiment (MICE) is being built at the ISIS $800 \mathrm{MeV}$ proton synchrotron at Rutherford Appleton Laboratory (RAL) in order to test ionization cooling of a muon beam. Successful demonstration of cooling is required in order to facilitate the creation of future high intensity muon beams in either a Neutrino Factory or Muon Collider. MICE should measure a $15 \%$ reduction in emittance of a muon beam with a precision of $1 \%$.

35th International Conference of High Energy Physics July 22-28, 2010

Paris, France

\footnotetext{
*Speaker.

On behalf of the MICE collaboration - http://mice.iit.edu
} 


\section{Neutrino Factory and Muon Collider}

Both Neutrino Factory and Muon Collider share the need of sufficient muon cooling channel in order to reduce the emittance of the produced muon beam.

\subsection{Neutrino Factory}

The Neutrino Factory, in which an intense, high-energy neutrino beam is produced from the decay of stored muon beams, has been proposed to serve a programme of precision measurements of neutrino oscillations. The Neutrino Factory has been shown to outperform second-generation superbeam and beta-beam facilities[1].

\subsection{Muon Collider}

Muons have negligible radiation and Beamstrahlung, so can be accelerated and stored in multirun recirculating devices. The liabilities of muons are that they decay, with a lifetime of $2.2 \times 10^{-6}$ $\mathrm{s}$ (in rest) and that they are created through decay into a spread phase space. The phase space can be reduced by ionization cooling, and lifetime could be increased enough for storige-ring collisions (e.g. at $2 \mathrm{TeV}$ muon lifetime is $0.044 \mathrm{~s}$ ). Following from the above a concept of muon collider, which accelerates and collides bunches of muons, can be introduced [2].

\section{MICE Schedule}

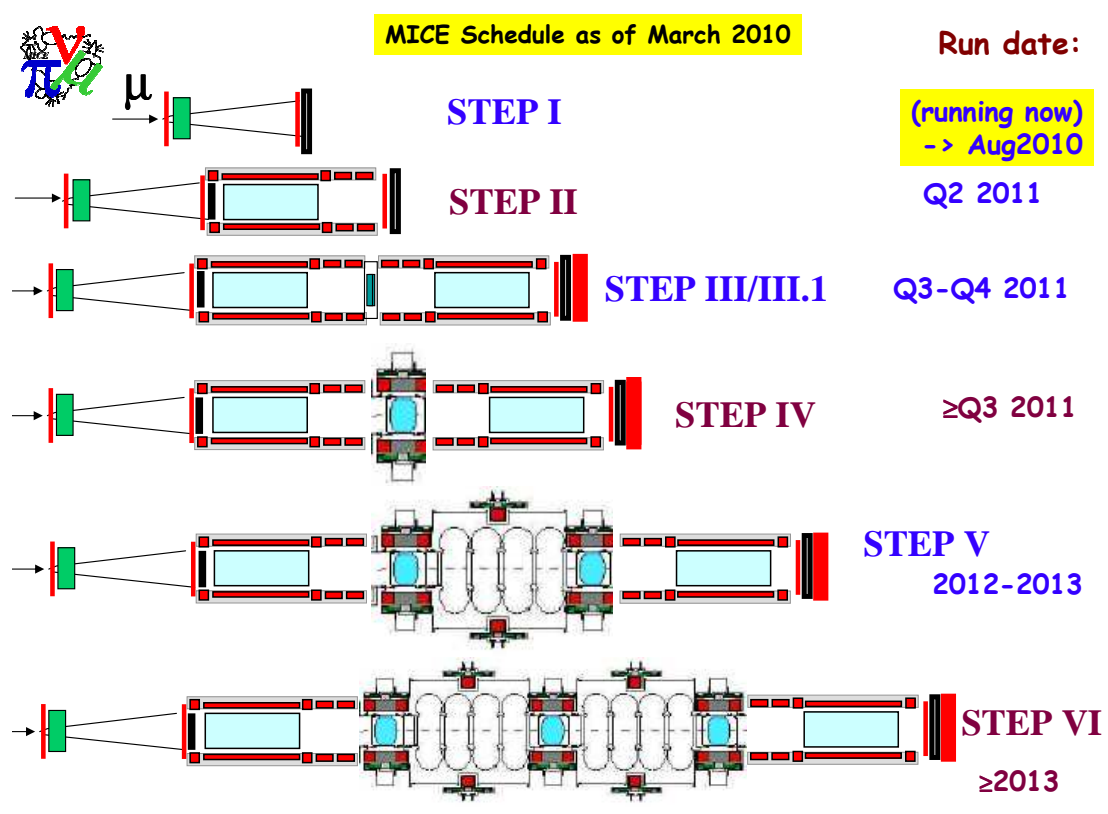

Figure 1: Muon ionization Cooling Experiment (MICE) Schedule. 
The International Muon Ionization Cooling Experiment was designed to feature 6 major steps [3]. In each step different measurements of the beamline and cooling channel are introduced (Figure 1).

\subsection{Step 1}

The first step of MICE is the establishment and precise measurement of a muon beam. The beamline consists of a titanium target, a set of dipole and quadrupole magnets to select and steer the beam, a solenoid to keep the pions focused while they decay, and a diffuser to generate the desired emittance. A set of particle identification detectors (Three Time-Of-Flight hodoscopes, two Cerenkov light counters and KLOE Light Calorimeter)[4] are used to distinguish between muons and other particles. First beam was observed in March 2008. All of the beamline components are operating according to specifications. Commissioning of the beamline and calibration of the PID detectors was completed in August 2010 [5].

\subsection{Step 2}

In this step the first spectrometer solenoid will be introduced allowing the first measurement of $6 \mathrm{D}$ emittance with high precision and comparison with the beam simulation. This should allow a systematic study of the tracker performance. The tracker has 5 stations with 3 planes of $350 \mathrm{~mm}$ fiber doublets in order to accurately allocate a point in space. MICE has two identical (upstream and downstream) trackers. Both trackers have been built and tested with cosmic rays. Each tracker will be immersed in a uniform $4 \mathrm{~T}$ field provided by the superconducting spectrometer solenoid magnets. An Electron-Muon Ranger (EMR) calorimeter made of 40 layers of extruded plastic scintillators will be added downstream to complement KL calorimeter in electron/muon selection as well as for additional tracking.

\subsection{Step 3}

Fundamental step for the understanding of a broad class of systematic errors in MICE. The two spectrometers will work together without any cooling device in between and should measure the same emittance value (up to the small predicted bias due to scattering in the spectrometers material). Characterization of solid state absorbers could be performed in this step.

\subsection{Step 4}

One focusing pair between the two spectrometers, should give a first experience with the operation of the absorber and a precise understanding of energy loss and multiple scattering in it. Several experiments with varying beta-functions and momentum as well as different absorbers can be performed with observation of transverse and longitudinal cooling.

\subsection{Step 5}

Starting from this step, the real goal of MICE, which is to establish the performance of a realistic cooling channel will be complete. Eight 201.25 MHZ RF (pill box) cavities with beryllium windows will be installed in the cooling section of the experiment, allowing up to $8 \mathrm{MV} / \mathrm{m}$ acceleration. 


\subsection{Step 6}

The final configuration of MICE includes a complete cell of the cooling channel. Only the central one of the three absorbers is fully representative of the SFOFO-lattice optics, since the other two must be run so as to match out of or into the spectrometers. The full Step VI configuration will also introduce the flexibility to simulate a variety of proposed lattice designs, including the "no-flip" lattice proposed at CERN.

\section{References}

[1] A. Blondel et al., "The Neutrino Factory: Beam and Experiments," Nucl. Instrum. Meth. A 451, 102 (2000).

[2] D. V. Neuffer and R. B. Palmer, "A high-energy high-luminosity $\mu^{+} \mu^{-}$collider," EPAC04-London, pp. 52-54, Jun. 1994

[3] The International Muon Ionization Cooling Experiment (MICE) proposal to the Rutherford Appleton Laboratory, 10 January 2003.

[4] M. Bonesini et al., "The MICE PID Instrumentation," 10th International Workshop on Neutrino Factories, Super beams and Beta beams, June 30 - July 5 2008, Valencia, Spain

[5] Y. Karadzhov "Status of MICE, the International Muon Ionisation Cooling Experiment," these proceedings 